\title{
Naturally-occurring fusidic acid resistance in staphylococci and its linkage to other resistances
}

\author{
R. J. EVANS AND PAMELA M. WATERWORTH \\ From the North West Surrey Group Laboratory, Chertsey, Surrey, \\ and the Postgraduate Medical School of London
}

SYNOPSIS Although Staph. aureus resistant to fusidic acid can be selected in vitro and has been found in vivo following treatment, naturally occurring strains without previous exposure to the drug have not, so far, been reported. Four such strains were isolated in the area of the North West Surrey Group Laboratory between August and September 1965, and six at Hammersmith Hospital during the first four months of 1966. In two of these resistance to fusidic acid was very unstable and its loss was accompanied by loss of resistance to penicillin in the first and to tetracycline and kanamycin in the second, although this strain remained resistant to penicillin.

If the sensitivity of Staphylococcus aureus to fusidic acid is tested by the broth dilution method, there may be as much as a 64-fold difference in the minimum inhibitory concentration according to the inoculum used. This has been shown to be due to the presence in any strain of a small number of resistant mutants (Godtfredsen, Roholt, and Tybring, 1962; Barber and Waterworth, 1962; Hilson, 1962; Taylor and Bloor, 1962). These organisms are at first uniformly resistant on subculture but may slowly become sensitive again after repeated transfer on normal medium.

In view of these observations it was predicted that resistance would emerge quickly in vivo, and both Lowbury, Cason, Jackson, and Miller (1962) and Newman, Bhat, Hackney, Robinson, and Stewart (1962a) reported the appearance of resistant staphylococci following treatment with fusidic acid. Nevertheless this has not been as common as anticipated; Taylor and Bloor (1962), Porter and Wilson (1963), and Dodson (1963) found no resistant staphylococci during the treatment of eight, 16, and 21 patients respectively, and Crosbie (1963) isolated resistant organisms from only one out of $\mathbf{2 0}$ cases of staphylococcal infection. To date no resistant strains have been reported without prior exposure to the drug, and the appearance of several in the area of the North West Surrey Group in the autumn of 1965 seemed of interest and stimulated us to investigate the subject and to search for further strains.

Received for publication 20 July 1966.

\section{MATERIALS AND METHODS}

All the staphylococci referred to were coagulase positive and were isolated from routine clinical specimens or from nasal swabs taken during cross-infection studies. At the North West Surrey Group Laboratory resistant strains were identified by disc sensitivity tests on primary cultures on blood agar or on subcultures on the same medium (discs containing $10 \mu \mathrm{g}$. fusidic acid); strains were maintained on agar slopes at $4^{\circ} \mathrm{C}$. and phage-typed by the Cross Infection Reference Laboratory, Colindale.

At Hammersmith primary sensitivity tests were done by pooling several colonies from the primary culture and streaking across agar plates with a lateral ditch containing $5 \mu \mathrm{g}$./ml. fusidic acid. Strains were maintained on Dorset egg slopes at room temperature and phage-typed in the Department.

All subsequent experiments were done with Oxoid no. 2 broth with or without $1.3 \%$ added agar, and diffusion sensitivities were done by one of the above methods. Fusidic acid is heavily protein-bound and inhibition zones are reduced by the presence of blood.

Incubation was at $37^{\circ} \mathrm{C}$. overnight except for the viable counts when it was continued for $\mathbf{4 8}$ hours.

\section{FUSIDIC ACID-RESISTANT STRAINS IN THE NORTH WEST} SURREY GROUP

Detailed records have been kept of some 8,000 strains of staphylococci isolated in this area since 1959, including their sensitivity to a variety of antibiotics selected in accordance with prevailing fashion. Fusidic acid was included in routine sensitivity test- 
ing in March 1964, since when some 2,700 isolates have been examined.

Resistance was first seen in August 1965 when a type $7 / 47 / 54 / 75$, resistant also to penicillin, was isolated from a patient (J.G.) suffering from chronic otitis media and under the care of a local practitioner. In the same month an untypable strain, resistant also to penicillin and tetracycline, was isolated from a paronychia in a patient (M.W.) under the care of another local practitioner. In October a type $7 / 47 / 54 / 75$, resistant also to penicillin and tetracycline, was isolated from the wound of a patient (F.S.) in St. Peter's Hospital, Chertsey, following an abdomino-perineal excision of the rectum, and in November a type $47 / 53 / 54 / 83 \mathrm{~A} /+$, resistant also to penicillin, was isolated from a post-operative wound infection in a patient (M.T.) in Woking Victoria Hospital.

In each case enquiries confirmed that no fusidic acid had been administered either in the present illness or on a previous occasion. The properties of the strains are summarized in Table I.

\section{FUSIDIC ACID-RESISTANT STRAINS AT HAMMERSMITH HOSPITAL}

Until the end of May 1965 fusidic acid was one of 10 antibiotics routinely tested against all staphylococci; it was then omitted as little was being used in the hospital and resistance to it was almost unknown. It was re-introduced in December 1965.

In January 1966 a type 6/42E/47/53/54/83/75/77/ $81+$, resistant to fusidic acid, penicillin, tetracycline, and kanamycin, was isolated from an ulcerated epithelioma of the leg in an out-patient (W.W.) who had attended the Radiotherapy Department. En:quiry revealed that a staphylococcus of the sames? phage type (and still in existence) had been isolated from this lesion in March 1965, when it was resistant to fusidic acid, penicillin, and tetracycline. Thes patient had been treated with polybactrin almos continuously between these dates.

In February a type $80+$, resistant also to penicil ${ }^{-}$ lin, was isolated from the nose of a 5-day-old infan $\vec{P}$ (M.H.); he had been delivered in the Hospital and went home briefly before being re-admitted because of fits. His mother was found to be a nasal carrier of type 29 , sensitive to fusidic acid.

During the next two months types $29 / 52 / 52 \mathrm{~A} / 79$ $80,81 / 7 / 47 / 77$, and $6 / 47 / 75$ were isolated fromb routine nasal swabs from a nurse (N.A.), a doctoks (D.M.), and a patient (I.T.) respectively; the first of these was resistant only to fusidic acid, the othersalso to penicillin.

In April a type 6/7/42E, resistant only to fusidic acid, was isolated from a post-operative woun infection in a patient (D.W.) whose pre-operative nasal swab had yielded a heavy growth of a type 75 , sensitive to fusidic acid and resistant to penicillin angु tetracycline.

None of these people had received fusidic acid af any time and there was no connexion between anys of them.

These strains are summarized in Table I.

\section{INSTABILITY OF RESISTANCE TO FUSIDIC ACID}

When the sensitivity of the strains isolated in the North West Surrey Group was checked after arp interval of three months, it was found that one (F.S. F)

TABLE I

CHARACTERISTICS OF FUSIDIC ACID-RESISTANT STRAINS OF Staph. aureus ISOLATED FROM PATIENTS NOT EXPOSED TO FUSIDIC ACID

$\begin{array}{ccc}\text { Hospital Date Source Phage Type } & \begin{array}{l}\text { Resistance Antibiotic Resistances } \\ \text { to Mercury }\end{array}\end{array}$

\begin{tabular}{|c|c|c|c|c|c|c|}
\hline J.G. & Local & Aug. 1965 & Ear & $7 / 47 / 54 / 75$ & $\mathbf{S}$ & Penicillin, fusidic acid \\
\hline M.T. & $\begin{array}{l}\text { Woking, Victoria } \\
\text { Hospital }\end{array}$ & Nov. 1965 & Wound & $47 / 53 / 54 / 83 \mathrm{~A} /+$ & $\mathbf{S}$ & Penicillin, fusidic acid \\
\hline M.W. & $\begin{array}{l}\text { Local } \\
\text { practitioner B }\end{array}$ & Aug. 1965 & Paronychia & Untypable & $\mathbf{R}$ & $\begin{array}{l}\text { Penicillin, fusidic acid, tetra- } \\
\text { cycline }\end{array}$ \\
\hline F.S. & $\begin{array}{l}\text { St. Peter's, } \\
\text { Chertsey }\end{array}$ & Oct. 1965 & Wound & $7 / 47 / 54 / 7 \mathrm{~B}$ & $\mathbf{S}$ & $\begin{array}{l}\text { Penicillin, fusidic acid, tetra- } \\
\text { cycline }\end{array}$ \\
\hline W.W.1 & Hammersmith & Mar. 1965 & Wound & $6 / 42 \mathrm{E} / 47 / 53 / 54 / 83 / 75 / 77 / 81+$ & $\mathbf{S}$ & $\begin{array}{l}\text { Penicillin, fusidic acid, tetra- } \\
\text { cycline }\end{array}$ \\
\hline W.W.2 & Hammersmith & Jan. 1966 & Wound & $6 / 42 \mathrm{E} / 47 / 53 / 54 / 83 / 75 / 77 / 81+$ & $\mathbf{S}$ & $\begin{array}{l}\text { Penicillin, fusidic acid, tetra- } \\
\text { cycline, kanamycin }\end{array}$ \\
\hline $\begin{array}{l}\text { M.H. } \\
\text { N.A. } \\
\text { D.M. } \\
\text { I.T. } \\
\text { D.W. }\end{array}$ & $\begin{array}{l}\text { Hammersmith } \\
\text { Hammersmith } \\
\text { Hammersmith } \\
\text { Hammersmith } \\
\text { Hammersmith }\end{array}$ & $\begin{array}{l}\text { Feb. } 1966 \\
\text { Feb. } 1966 \\
\text { Mar. } 1966 \\
\text { Apr. } 1966 \\
\text { Apr. } 1966\end{array}$ & $\begin{array}{l}\text { Nose } \\
\text { Nose } \\
\text { Nose } \\
\text { Nose } \\
\text { Wound }\end{array}$ & $\begin{array}{l}80+ \\
29 / 52 / 52 \mathrm{~A} / 79 / 80 \\
81 / 7 / 47 / 77 \\
6 / 47 / 75 \\
6 / 7 / 42 \mathrm{E}\end{array}$ & $\begin{array}{l}\mathbf{S} \\
\mathbf{S} \\
\mathbf{S} \\
\mathbf{S} \\
\mathbf{S}\end{array}$ & $\begin{array}{l}\text { Penicillin, fusidic acid } \\
\text { Fusidic acid } \\
\text { Penicillin, fusidic acid } \\
\text { Penicillin, fusidic acid } \\
\text { Fusidic acid }\end{array}$ \\
\hline
\end{tabular}


strain (J.G.) heterogeneous in resistance, were counted by the Miles and Misra technique on plates containing serial dilutions of fusidic acid. The results given in Table II show that although the culture (J.G.) contains some cells much more resistant than any in the control culture, the greater part of the population is fully sensitive. This was not the case with the resistant strain (M.W.) in which the majority of the cells are uniformly resistant.

\section{INVESTIGATION OF CULTURES CONTAINING SENSITIVE AND RESISTANT COLONIES}

Resistant colonies from either J.G. or W.W. at first yielded uniformly resistant cultures but these rapidly produced sensitive variants if serially subcultured on normal medium and the resulting cultures were then indistinguishable from those of the parents. Conversely, serial subcultures of sensitive colonies led not to the reproduction of the original mixed population but to cultures which remained uniformly sensitive. It was also found that the sensitive variant from J.G. had lost its resistance not only to fusidic acid but also to penicillin, and that the sensitive variant from W.W. was now sensitive to tetracycline and kanamycin as well as to fusidic acid and resistant only to penicillin. The sensitive and resistant variants were phage-typed and found to be identical with each other and with their parent culture.

Repeated subculture of the resistant variants on normal medium rapidly reduced the number of resistant organisms present but those remaining kept their original multiple resistance pattern. This was confirmed by the replica plating of about 500 colonies from each strain on to plates containing the relevant antibiotics. All colonies from J.G. were sensitive either to penicillin and fusidic acid or to neither, and those from W.W. were resistant to peni- cillin alone or to penicillin, tetracycline, kanamycin, and fusidic acid.

DEVELOPMENT OF RESISTANCE TO FUSIDIC ACID In Vitro

Volumes of broth, each of $1 \mathrm{ml}$., containing serial dilutions of fusidic acid were inoculated with $0.02 \mathrm{ml}$. overnight broth cultures of the sensitive variants of J.G. or W.W. diluted $1 / 500(a)$ and undiluted $(b) . \vec{\circ}$ After overnight incubation all the tubes with the heavy inoculum $(b)$ were streaked across ditch plates $\omega$ containing fusidic acid, penicillin and, in the case of W.W., tetracycline.

The results are given in Table III. All dilutions $\vec{\oplus}$ showing growth only from a heavy inoculum yielded $c$ organisms resistant to fusidic acid but survivors from tubes showing no growth remained sensitive, as dido the bulk of the population in tubes immediately below the minimum inhibitory concentration for az small inoculum.

None of the fusidic acid-resistant cultures showed resistance to the other antibiotics included in the ditch plates; their behaviour was identical with that of a sensitive control staphylococcus tested in thes same way.

Nevertheless, a very heavy inoculum of the sensitive variant of W.W. (taken from solid growth) streaked across a kanamycin ditch yielded two colonies resistant also to fusidic acid and tetras cycline. A broth culture of this sensitive variant was then counted on plates containing serial dilutions of fusidic acid: $0.02 \mathrm{ml}$. undiluted culture yielded $10 \mathrm{~g}$ colonies on $2 \mu \mathrm{g} . / \mathrm{ml}$. and three colonies on $4 \mu \mathrm{g} . / \mathrm{ml}$.; two of the former possessed multiple resistance, the remainder were resistant only to fusidic acid.

No penicillin-resistant colonies could be obtained from the sensitive variant of J.G.

TABLE III

SENSITIVITY PATTERNS OF SENSITIVE VARIANTS AFTER EXPOSURE TO FUSIDIC ACID IN BROTH

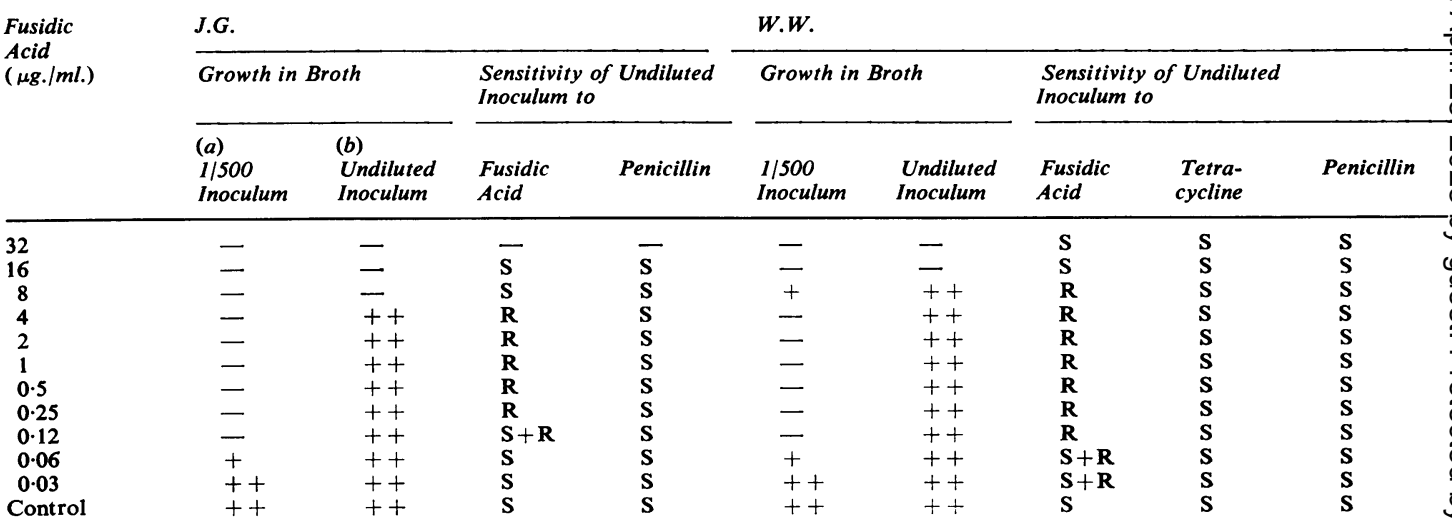




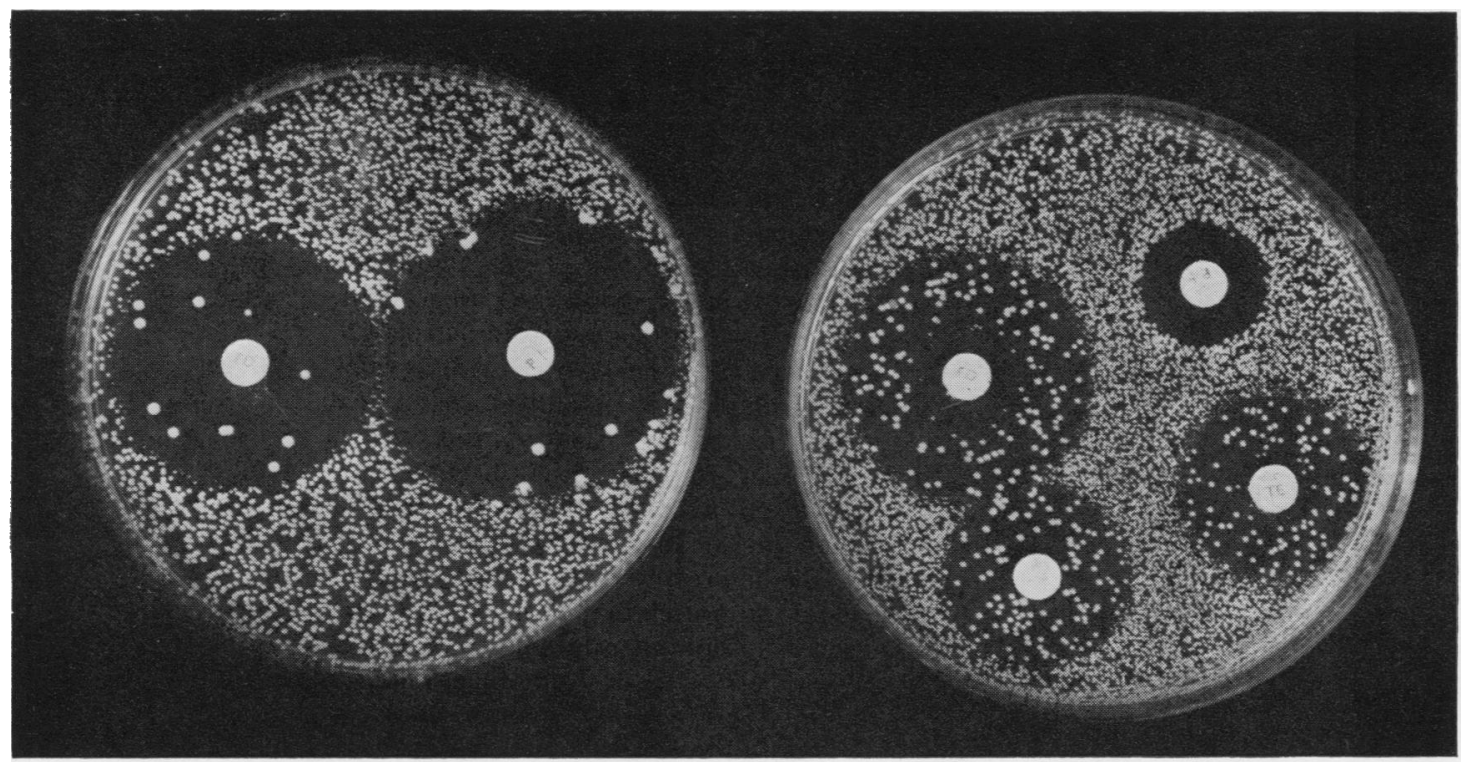

FIG. 2. Sensitivity tests by the disc method on cultures containing sensitive and resistant variants. Left: J.G. Right: W.W. Discs contain fusidic acid $10 \mu g$., tetracycline $10 \mu g$., penicillin 5 units, kanamycin $30 \mu g$.

\section{DISCUSSION}

These naturally occurring resistant strains resemble resistant variants obtained from sensitive cultures in vitro in their degree of resistance to fusidic acid, and in its rapid loss in two of them; but it seems likely that they originate in different ways.

It is known that penicillin resistance in naturallyoccurring, penicillina-seproducing Staph. aureus may be controlled by extra-chromosomal elements (plasmids) and that mercury resistance is commonly carried on the same plasmid (Novick, 1963; Harmon and Baldwin, 1964; Hashimoto, Kono, and Mitsuhashi, 1964). Hashimoto et al. (1964) also described one strain in which erythromycin resistance was similarly linked with penicillinase production. Novick and Richmond (1965), studying this strain, found both resistances to be co-eliminated or cotransduced at a frequency greater than $99 \%$, but were unable to find any more strains in which erythromycin and penicillin resistance were linked.

No transduction experiments have been done with the strains J.G. and W.W. described here, but the high rate of loss of resistance simultaneously to fusidic acid and penicillin from the former, and to fusidic acid, tetracycline, and kanamycin' from the latter, strongly suggests that these resistances are controlled by single plasmids. Resistance to penicillin in W.W. was more stable and may therefore be controlled by a separate stable plasmid, or as recent work by Asheshov (1966) had indicated, by a chromosome.

The development of resistance in vivo to kanamycin in the strain W.W. between March 1965 and January 1966 was presumably the result of treatment with a related drug (neomycin) during this period, but there is no evidence of how this occurred. It is remarkable that a further resistance should by added to an unstable plasmid already controlling resistance to both fusidic acid and tetracycline but, once it had occurred, treatment with neomycin would by selection ensure survival of the variant resistant to fusidic acid. It is also possible that the instability of resistance is due to the slower rate of growth of the plasmid in vitro than in vivo.

Fusidic acid-resistant mutants selected from a heavy inoculum of the sensitive variants of these strains do not regain the sensitivity pattern of the original isolates but behave as other resistant mutants selected from sensitive cultures in vitro.

Penicillin-resistant staphylococci did not become apparent until after the introduction of penicillin and in may be that naturally occurring resistance to fusidic acid exists and is only now appearing. As long ago as 1963 one of us (P.M.W.) examined a strain of Staph. aureus sent to the late Professor Mary Barber from Turkey and found it to have a similar degree of resistance to fusidic acid although this drug was not then available in that country. 
If a strain containing both sensitive and resistant variants is tested by the disc method with a light inoculum, it may easily be reported as sensitive; if tested on subculture only sensitive colonies may be selected. If such strains are common, resistance may be more frequent than is suspected; the sensitive variant of W.W. had only 1 in $5,500,000$ cells resistant to fusidic acid, tetracycline, and kanamycin but treatment with any of these drugs would select organisms resistant to them all.

\section{ADDENDUM}

Since this paper was completed, a further fusidic acid-resistant strain has been isolated in the Nortio West Surrey Group Laboratory.

\section{REFERENCES}

Asheshov, E. A. (1966). Nature (Lond.), 210, 804.

Barber, M., and Waterworth, P. M. (1962). Lancet, 1, 931.

Crosbie, R. B. (1963). Brit. med. J., 1, 788.

Dodson, B. (1963). Lancet, 2, 659.

Godtfredsen, W., Roholt, K., and Tybring, L. (1962). Ibid., 1, $928 \hat{9}$

Harmon, S. A., and Baldwin, J. N. (1964). J. Bact., 87, 593.

Hashimoto, H., Kono, K., and Mitsuhashi, S. (1964). Ibid., 88, 261.

Hilson, G. R. F. (1962). Lancet, 1, 932.

Lowbury, E. J. L., Cason, J. S., Jackson, D.MacG., and Millexw R. W. S. (1962). Ibid., 2, 478. Newman, R. L., Bhat, K. M., Hackney, R., Robinson, C., anक़
Stewart, G. T. (1962). Brit. med. J., 2, 1645.

Novick, R. P. (1963). J. gen. Microbiol., 33, 121.

Novick, R. P., and Richmond, M. H. (1965). J. Bact., 90, 467.

Porter, I. A., and Wilson, J. S. P. (1963). Lancet, 2, 658.

Taylor, G., and Bloor, K. (1962). Ibid., 1, 935. 